\title{
STEPANOV'S METHOD APPLIED TO BINOMIAL EXPONENTIAL SUMS
}

\author{
TODD COCHRANE AND CHRISTOPHER PINNER
}

\begin{abstract}
For a prime $p$ and binomial $a x^{k}+b x^{l}$ with $1 \leq l<k<\frac{1}{32}(p-1)^{\frac{2}{3}}$, we use Stepanov's method to obtain the bound

$$
\left|\sum_{x=1}^{p-1} e_{p}\left(a x^{k}+b x^{l}\right)\right| \ll \max \left\{1, l \Delta^{-\frac{1}{3}}\right\}^{\frac{1}{4}} k^{\frac{1}{4}} p^{\frac{3}{4}},
$$

where $\Delta=\frac{k-l}{(k, l, p-1)}$.
\end{abstract}

\section{IntRoduction}

For a prime $p$ and binomial $f(x)=a x^{k}+b x^{l}$ we consider the binomial exponential sum

$$
S^{*}=\sum_{x=1}^{p-1} e_{p}\left(a x^{k}+b x^{l}\right), \quad p \nmid a b, \quad 1 \leq l<k<p-1,
$$

where $e_{p}(\cdot)$ is the additive character $e_{p}(\cdot)=e^{2 \pi i \cdot / p}$ on the finite field $\mathbb{Z}_{p}$. For such sums the classical Weil bound [9]

$$
\left|S^{*}\right| \leq(k-1) p^{\frac{1}{2}}
$$

is non-trivial as long as $k<p^{\frac{1}{2}}$. When $l$ is significantly smaller than $k$ a bound of Mordell [7] as refined in [2] can extend this range:

$$
\left|S^{*}\right|<(k l)^{\frac{1}{4}} p^{\frac{3}{4}}
$$

a bound also obtained in the special case $l=1$ by Karatsuba [6]. For monomial sums Heath-Brown \& Konyagin [5] successfully used Stepanov's method to obtain a bound non-trivial for $k \leq p^{\frac{2}{3}}$

$$
\left|\sum_{x=1}^{p-1} e_{p}\left(a x^{k}\right)\right| \ll \min \left\{k^{\frac{5}{8}} p^{\frac{5}{8}}, k^{\frac{3}{8}} p^{\frac{3}{4}}\right\},
$$

improving upon the Weil bound (1.2) once $k>p^{\frac{1}{3}}$. Konyagin informs us that he has made additional refinements, further extending this range. We show here how Stepanov's

Date: September 8, 2009.

1991 Mathematics Subject Classification. 11L07;11L03.

Key words and phrases. exponential sums. 
method can be similarly used for binomials to improve the Mordell and Weil bounds when $k<\frac{1}{32}(p-1)^{\frac{2}{3}}$. We are able to deal with the slightly more general sums

$$
S_{+}=\sum_{x=1}^{p-1} \chi(x) e_{p}\left(a x^{k}+b x^{l}\right), \quad p \nmid a b, \quad 1 \leq l<k<p-1,
$$

and

$$
S_{-}=\sum_{x=1}^{p-1} \chi(x) e_{p}\left(a x^{k}+b x^{-l}\right), \quad p \nmid a b, \quad 1 \leq l \leq k, \quad(k+l)<p-1,
$$

where $\chi(x)$ is a multiplicative character $\bmod p$. For these generalized sums the Weil bound (see [8]) still gives

$$
\left|S_{+}\right| \leq k p^{\frac{1}{2}}, \quad\left|S_{-}\right| \leq(k+l) p^{\frac{1}{2}}
$$

and the Mordell type bound (see [2]),

$$
\left|S_{+}\right|<(k l)^{\frac{1}{4}} p^{\frac{3}{4}}, \quad\left|S_{-}\right|<(3 k l)^{\frac{1}{4}} p^{\frac{3}{4}} .
$$

We write

$$
d=(l, k, p-1),
$$

and

$$
\Delta_{+}=\frac{(k-l)}{d}, \quad \Delta_{-}=\frac{(k+l)}{d}
$$

Theorem 1.1. For $k<\frac{1}{32}(p-1)^{\frac{2}{3}} d^{\frac{1}{3}}$,

$$
\left|S_{ \pm}\right| \ll \max \left\{1, l \Delta_{ \pm}^{-\frac{1}{3}}\right\}^{\frac{1}{4}} k^{\frac{1}{4}} p^{\frac{3}{4}}
$$

Notice that when $k \ll p^{\frac{2}{3}} d^{\frac{1}{3}}$ and Theorem 1.1 applies, our bound (1.11) is plainly never worse in order than the Mordell type bound (1.3), improving on it once $l \gg 1$ and $k-l \gg d$. One particular feature of our result is that we do not need the restrictions $k \mid(p-1)$ and $(k, l)=1$ that appear in the binomial bounds (1.14) and (1.15) of Akulinicev [1] and $\mathrm{Yu}$ [10]. As a special case, when $l<(k / d)^{\frac{1}{3}}$ we observe that Theorem 1.1 simplifies to $\left|S_{ \pm}\right| \ll k^{\frac{1}{4}} p^{\frac{3}{4}}$, always non-trivial and surpassing the Weil bound (1.2) when $p^{\frac{1}{3}} \ll k \ll p^{\frac{2}{3}}$, the same range as for the Heath-Brown \& Konyagin monomial result (1.4).

In $[2$, Theorem 1.2] we showed that

$$
\left|S_{ \pm}\right| \leq p^{\frac{1}{4}} M_{ \pm}^{\frac{1}{4}}
$$

where

$$
M_{+}=\#\left\{\left(x_{1}, x_{2}, x_{3}, x_{4}\right) \in \mathbb{Z}_{p}^{* 4}: x_{1}^{k}+x_{2}^{k}=x_{3}^{k}+x_{4}^{k}, x_{1}^{l}+x_{2}^{l}=x_{3}^{l}+x_{4}^{l}\right\},
$$


and

$$
M_{-}=\#\left\{\left(x_{1}, x_{2}, x_{3}, x_{4}\right) \in \mathbb{Z}_{p}^{* 4}: x_{1}^{k}+x_{2}^{k}=x_{3}^{k}+x_{4}^{k}, x_{1}^{-l}+x_{2}^{-l}=x_{3}^{-l}+x_{4}^{-l}\right\},
$$

with (1.8) corresponding to the straightforward bounds

$$
M_{+}<k l(p-1)^{2}, \quad M_{-}<3 k l(p-1)^{2} .
$$

Hence Theorem 1.1 amounts to showing that in certain cases we can improve these bounds:

Lemma 1.1. For $k<\frac{1}{32}(p-1)^{\frac{2}{3}} d^{\frac{1}{3}}$

$$
M_{ \pm} \ll \max \left\{1, l \Delta_{ \pm}^{-\frac{1}{3}}\right\} k p^{2} .
$$

This result is perhaps initially surprising since there are certainly situations in which (1.13) can not be improved; for example when $k=2 l, l \mid(p-1)$ it is readily verified that $M_{+}=2 l^{2}(p-1)^{2}-l^{3}(p-1)$, and when $k=l$ and $2 l \mid(p-1)$ that $M_{-}=3 l^{2}(p-1)^{2}-3 l^{3}(p-1)$, so that $M_{ \pm}=O\left(k p^{2}\right)$ is not in general true. The proof of Lemma 1.1 occupies sections 2, 3 and 4. Theorem 1.1 follows immediately from Lemma 1.1 and (1.12).

There are two parameters that must be controlled in order to expect a uniform nontrivial bound on $\left|S_{ \pm}\right|$for arbitrary $f$ with given exponents $k, l$. The first is $d=(k, l, p-1)$. In [3, Corollary 2.1] the authors showed that for given $1<l<k$ with $d>\frac{p}{\log p}$ there exist $a, b$ such that $\left|S^{*}\right|$ is very close to $p$ in size; to be precise $\left|S^{*}\right|>p\left(1-\frac{2 \pi^{2}}{\left\lfloor p^{2 d /(p-1)}\right\rfloor^{2}}\right)-1$. The second parameter is the quantity

$$
d_{+}^{*}=(k-l, p-1), \quad d_{-}^{*}=(k+l, p-1) .
$$

The following theorem shows that there is always a collection of exceptional sums where $\left|S_{ \pm}\right| \sim d_{ \pm}^{*}$, but that aside from these exceptions there is a uniform nontrivial bound when $d_{ \pm}^{*}$ is large:

Proposition 1.1. a) If $\chi^{(p-1) / d_{ \pm}^{*}}=\chi_{0}$ and $-b \bar{a}=\alpha^{(k \mp l)}$ for some $\alpha$ in $\mathbb{Z}_{p}^{*}$, then

$$
\left|S_{ \pm}-\chi(\alpha) d_{ \pm}^{*}\right| \leq\left(p-1-d_{ \pm}^{*}\right) \frac{d}{d_{ \pm}^{*}} p^{\frac{1}{2}}
$$

b) Otherwise, we have uniformly

$$
\left|S_{ \pm}\right| \leq \frac{d}{d_{ \pm}^{*}} p^{\frac{3}{2}}
$$

Though the bound as stated is only non-trivial when $d_{ \pm}^{*}>d p^{\frac{1}{2}}$, by using (1.4) instead of the Weil bound in (5.1), one can replace the right-hand side of both inequalities by

$$
O\left(\min \left\{\left(d / d_{ \pm}^{*}\right)^{\frac{5}{8}} p^{\frac{5}{4}},\left(d / d_{ \pm}^{*}\right)^{\frac{3}{8}} p^{\frac{9}{8}}\right\}\right),
$$

extending this range to $d_{ \pm}^{*} \gg d p^{\frac{1}{3}}$ and improving the bound when $d_{ \pm}^{*} \ll d p^{\frac{2}{3}}$. 
We deduce from Theorem 1.1 and Proposition 1.1 the following uniform upper bound on $S_{ \pm}$, nontrivial for $d<c p^{1 / 12}$, $c$ a sufficiently small constant.

Corollary 1.1. a) If $S_{ \pm}$is not one of the exceptional sums in part (a) of Proposition 1.1 or $d_{ \pm}^{*} \leq d^{\frac{3}{13}} p^{\frac{51}{52}}$ then we have uniformly

$$
\left|S_{ \pm}\right| \ll d^{3 / 13} p^{51 / 52}
$$

b) If $S_{ \pm}$is one of the exceptional sums in part (a) of Proposition 1.1 and $d_{ \pm}^{*}>d^{\frac{3}{13}} p^{\frac{51}{52}}$ then

$$
\left|S_{ \pm}-\chi(\alpha) d_{ \pm}^{*}\right| \ll d^{10 / 13} p^{27 / 52}
$$

In particular, we see that if $\chi$ is the trivial character or Legendre symbol and

$$
f(x)=a\left(x^{\frac{1}{2}(p-1)}-\alpha^{\frac{1}{2}(p-1)}\right) x^{ \pm l}
$$

for some $\alpha$ in $\mathbb{Z}_{p}^{*}$ and $1 \leq l<\frac{1}{2}(p-1)$, then

$$
\left|S_{ \pm}-\frac{\chi(\alpha)}{2}(p-1)\right| \leq d p^{\frac{1}{2}}
$$

For all remaining cases, if $d<c p^{\frac{1}{12}}$ with $c$ a sufficiently small constant,

$$
\left|S_{ \pm}\right| \leq \frac{1}{3}(p-1)+2 d \sqrt{p}
$$

In comparison, for binomials with $k \mid(p-1)$ and $(k, l)=1$ Akulinicev [1] obtained the bound

$$
\left|S^{*}\right| \leq \frac{p}{\sqrt{k}}+\sqrt{(l, p-1)-1} p^{\frac{3}{4}}
$$

and deduced when $l=1$ and $k \mid(p-1)$ the uniform bound

$$
\left|S^{*}\right| \leq p^{\frac{5}{6}}
$$

$\mathrm{Yu}[10]$ obtained

$$
\left|S^{*}\right| \ll k^{-\frac{3}{8}} p^{\frac{9}{8}}
$$

and hence the uniform bound

$$
\left|S^{*}\right| \ll p^{\frac{21}{22}}
$$

under the weaker assumptions $k \mid(p-1)$ and $(k, l)=1$. 
2. Initial DECOMPOSITION OF $M_{ \pm}$

We write

$$
M_{ \pm}=\sum_{0 \leq u_{1}, u_{2} \leq p-1} R_{ \pm}\left(u_{1}, u_{2}\right)^{2}
$$

where

$$
R_{ \pm}\left(u_{1}, u_{2}\right)=\#\left\{(x, y) \in \mathbb{Z}_{p}^{* 2}: x^{k}-y^{k}=u_{1}, x^{ \pm l}-y^{ \pm l}=u_{2}\right\}
$$

Plainly

$$
R_{ \pm}(0,0)=\#\left\{(x, y) \in \mathbb{Z}_{p}^{* 2}:(x \bar{y})^{k}=1,(x \bar{y})^{ \pm l}=1\right\}=d(p-1)
$$

When $\left(u_{1}, u_{2}\right) \neq(0,0)$ we always have the trivial bound

$$
R_{+}\left(u_{1}, u_{2}\right) \leq(k-d) l
$$

since $x$ must be a root of the non-zero polynomial

$$
\left(x^{k}-u_{1}\right)^{l / d}-\left(x^{l}-u_{2}\right)^{k / d}=0
$$

of degree at most $(k / d-1) l$ and $y^{(l, k)}=\left(x^{k}-u_{1}\right)^{s}\left(x^{l}-u_{2}\right)^{t}$ where $k s+l t=(l, k)$. For $M_{-}$we have $\left(x^{k}-u_{1}\right)^{l / d}\left(u_{2} x^{l}-1\right)^{k / d}-(-1)^{k / d} x^{k l / d}=0$ and $R_{-}\left(u_{1}, u_{2}\right) \leq 2 k l$. Hence, writing

$$
\ell_{+}=l, \quad \ell_{-}=2 l,
$$

we have

$$
\sum_{u_{1}=1}^{p-1} R_{ \pm}^{2}\left(u_{1}, 0\right)<k \ell_{ \pm} \sum_{u_{1}=0}^{p-1} R_{ \pm}\left(u_{1}, 0\right)=k \ell_{ \pm} \#\left\{(x, y) \in \mathbb{Z}_{p}^{* 2}: x^{ \pm l}=y^{ \pm l}\right\} \leq l(p-1) k \ell_{ \pm}
$$

Likewise $\sum_{u_{2}=1}^{p-1} R_{ \pm}^{2}\left(0, u_{2}\right)<k^{2} \ell_{ \pm}(p-1)$. For the $u_{1}, u_{2} \neq 0$ we write $R_{ \pm}\left(u_{1}, u_{2}\right)=$ $d C_{ \pm}\left(u_{1}, u_{2}\right)$ where

$$
C_{ \pm}\left(u_{1}, u_{2}\right)=\#\left\{x \in \mathbb{Z}_{p}^{*}: x^{k}-1=u_{1} y^{k}, x^{ \pm l}-1=u_{2} y^{ \pm l} \text { for some } y \in \mathbb{Z}_{p}^{*}\right\}
$$

to see this observe that counting the number of $(x, y)$ satisfying $(x \bar{y})^{k}-1=u_{1} \bar{y}^{k},(x \bar{y})^{ \pm l}-$ $1=u_{2} \bar{y}^{ \pm l}$, is the same as counting the $(x, y)$ satisfying $x^{k}-1=u_{1} y^{k}, x^{ \pm l}-1=u_{2} y^{ \pm l}$, where $y^{(k, l)}=\left(x^{k}-1\right)^{s}\left(x^{ \pm l}-1\right)^{t}{\overline{u_{1}}}^{s}{\overline{u_{2}}}^{t}, k s \pm l t=(k, l)$ shows that there will be exactly $d$ different $y$ for each $x$ with a solution. Clearly if $u_{1}=u^{k} \tilde{u}_{1}$ then $u_{1} y^{k}=\tilde{u}_{1}(u y)^{k}$, $u_{2} y^{ \pm l}=u_{2} \bar{u}^{ \pm l}(u y)^{ \pm l}$ and $C_{ \pm}\left(u_{1}, u_{2}\right)=C_{ \pm}\left(\tilde{u}_{1}, u_{2} \bar{u}^{ \pm l}\right)$, while if $u_{2}=u^{ \pm l} \tilde{u}_{2}$ with $u^{k}=1$ then $u_{1} y^{k}=u_{1}(u y)^{k}, u_{2} y^{ \pm l}=\tilde{u}_{2}(u y)^{ \pm l}$ and $C_{ \pm}\left(u_{1}, u_{2}\right)=C_{ \pm}\left(u_{1}, \tilde{u}_{2}\right)$. Hence, using $\alpha$ to 
denote a primitive root $\bmod p$, and writing $k_{1}=(k, p-1)$,

$$
\begin{aligned}
\sum_{1 \leq u_{1}, u_{2} \leq p-1} R_{ \pm}\left(u_{1}, u_{2}\right)^{2} & =d^{2} \sum_{u_{2}=1}^{p-1} \sum_{u_{1}=1}^{p-1} C_{ \pm}^{2}\left(u_{1}, u_{2}\right) \\
& =d^{2} \frac{p-1}{k_{1}} \sum_{u_{2}=1}^{p-1} \sum_{j=1}^{k_{1}} C_{ \pm}^{2}\left(\alpha^{j}, u_{2}\right)=d(p-1) \sum_{j=1}^{k_{1}} \sum_{i=1}^{\frac{(p-1) d}{k_{1}}} C_{ \pm}^{2}\left(\alpha^{j}, \alpha^{i}\right) .
\end{aligned}
$$

We show that for this latter sum no $x$ will be counted more than once: If

$$
x^{k}-1=\alpha^{j_{1}} y_{1}^{k}=\alpha^{j_{2}} y_{2}^{k}, \quad x^{ \pm l}-1=\alpha^{i_{1}} y_{1}^{ \pm l}=\alpha^{i_{2}} y_{2}^{ \pm l},
$$

for some $y_{1}, y_{2} \in \mathbb{Z}_{p}^{*}$ and $i_{1}, i_{2}, j_{1}, j_{2}$ with $1 \leq j_{1}, j_{2} \leq k_{1}$ and $1 \leq i_{1}, i_{2} \leq(p-1) d / k_{1}$, then $\alpha^{\left(j_{1}-j_{2}\right)}=\left(y_{2} \bar{y}_{1}\right)^{k}$ and $j_{1}=j_{2}$. Since $\left(y_{2} \bar{y}_{1}\right)^{k}=1$ we have $y_{2} \bar{y}_{1}=\alpha^{(p-1) u / k_{1}}$ for some $u$, and

$$
\alpha^{\left(i_{1}-i_{2}\right)}=\left(y_{2} \bar{y}_{1}\right)^{ \pm l}=\alpha^{ \pm \frac{(p-1)}{\left(k_{1} / d\right)} u(l / d)}
$$

forcing $(p-1) d / k_{1} \mid\left(i_{1}-i_{2}\right)$ and $i_{1}=i_{2}$.

For a pair $u_{1}, u_{2}$ in $\mathbb{Z}_{p}^{*}$ and corresponding vector $\mathbf{u}=\left(u_{1}, u_{2}\right)$ we shall sometimes write $C_{ \pm}(\mathbf{u})$ for $C_{ \pm}\left(u_{1}, u_{2}\right)$. We suppose that there are $N$ non-zero $C_{ \pm}\left(\alpha^{j}, \alpha^{i}\right)$, say $C_{ \pm}\left(\mathbf{u}_{1}\right), \ldots, C_{ \pm}\left(\mathbf{u}_{N}\right)$, as $i, j$ run through $1 \leq j \leq k_{1}, 1 \leq i \leq(p-1) d / k_{1}$ and order them so that

$$
C_{ \pm}\left(\mathbf{u}_{1}\right) \geq C_{ \pm}\left(\mathbf{u}_{2}\right) \geq \cdots \geq C_{ \pm}\left(\mathbf{u}_{N}\right)
$$

Hence

$$
M_{ \pm} \leq d^{2}(p-1)^{2}+2 k^{2} \ell_{ \pm}(p-1)+d(p-1) \sum_{i=1}^{N} C_{ \pm}^{2}\left(\mathbf{u}_{i}\right) .
$$

Also, since the sets defining the $C_{ \pm}\left(\mathbf{u}_{i}\right)$ are disjoint,

$$
\sum_{i=1}^{N} C_{ \pm}\left(\mathbf{u}_{i}\right) \leq(p-1)
$$

\section{Applying the Stepanov Method}

We write

$$
d_{1}=(l, k), \quad \delta_{+}=\frac{k-l}{d_{1}}, \quad \delta_{-}=\frac{k+l}{d_{1}}, \quad \ell_{+}=l, \quad \ell_{-}=2 l,
$$

and set

$$
T= \begin{cases}\left\lfloor 2^{-\frac{9}{2}}(p-1)\left(\frac{\delta_{ \pm}^{2}}{k \ell_{ \pm} / d_{1}}\right)^{-\frac{3}{2}}\right\rfloor, & \text { if }\left(k \ell_{ \pm} / d_{1}\right)<\frac{1}{2} \delta_{ \pm}^{2}, \\ \left\lfloor 2^{-7}(p-1)\left(\frac{\delta_{ \pm}^{2}}{k \ell_{ \pm} / d_{1}}\right)\right\rfloor, & \text { if }\left(k \ell_{ \pm} / d_{1}\right) \geq \frac{1}{2} \delta_{ \pm}^{2} .\end{cases}
$$


We use Stepanov's method to bound the size of the $C_{ \pm}\left(\mathbf{u}_{t}\right)$ for $t \leq T$. If $T>N$ we set $C_{ \pm}\left(\mathbf{u}_{t}\right)=0$ for the $N<t \leq T$.

Lemma 3.1. Suppose that $k<2^{-5}(p-1)^{\frac{2}{3}} d_{1}^{\frac{1}{6}} \ell_{ \pm}^{\frac{1}{6}}$. Then

$$
C_{ \pm}\left(\mathbf{u}_{t}\right) \leq 2^{\frac{26}{5}}(p-1)^{\frac{2}{5}}\left(k \ell_{ \pm} / d_{1}\right)^{\frac{3}{5}} t^{-\frac{2}{5}} \delta_{ \pm}^{-\frac{1}{5}}, \quad 1 \leq t \leq T .
$$

In particular $C_{ \pm}\left(\mathbf{u}_{T}\right) \leq 2^{\frac{37}{5}} \delta_{ \pm}$when $\left(k \ell_{ \pm} / d_{1}\right)<\frac{1}{2} \delta_{ \pm}^{2}$.

Proof. If $x$ is counted in $C_{+}\left(u_{1}, u_{2}\right)$ so that $x^{k}-1=u_{1} y^{k}, x^{l}-1=u_{2} y^{l}$, for some $y \in \mathbb{Z}_{p}^{*}$, then

$$
\left(x^{k}-1\right)^{l / d_{1}} u_{2}^{k / d_{1}}-\left(x^{l}-1\right)^{k / d_{1}} u_{1}^{l / d_{1}}=0,
$$

while for $C_{-}\left(u_{1}, u_{2}\right)$ we have instead $x^{-l}-1=u_{2} y^{-l}$ and

$$
\left(x^{k}-1\right)^{l / d_{1}}\left(x^{l}-1\right)^{k / d_{1}}-(-1)^{k / d_{1}} u_{1}^{l / d_{1}} u_{2}^{k / d_{1}} x^{k l / d_{1}}=0 .
$$

Hence we have the trivial bounds

$$
C_{ \pm}(\mathbf{u}) \leq \min \left\{(p-1), k \ell_{ \pm} / d_{1}\right\}
$$

and may assume that

$$
t^{2 / 5}>2^{\frac{26}{5}} \frac{\left(k \ell_{ \pm} / d_{1}\right)^{\frac{3}{5}}}{\delta_{ \pm}^{\frac{1}{5}}(p-1)^{\frac{3}{5}}}, \quad t^{2 / 5}>2^{\frac{26}{5}} \frac{(p-1)^{\frac{2}{5}}}{\left(k \ell_{ \pm} / d_{1}\right)^{\frac{2}{5}} \delta_{ \pm}^{\frac{1}{5}}}
$$

Consider the polynomials

$$
F(x)=\sum_{i<A} \Phi_{i}(x) f_{i}(x)
$$

with

$$
\Phi_{i}(x)=\left(x^{k}-1\right)^{(A-i) l / d_{1}}\left(x^{l}-1\right)^{i k / d_{1}}
$$

for $M_{+}$and

$$
\Phi_{i}(x)=\left(x^{k}-1\right)^{i l / d_{1}}\left(x^{l}-1\right)^{i k / d_{1}} x^{(A-i) k l / d_{1}}
$$

for $M_{-}$, and

$$
f_{i}(x)=\sum_{j<B} \sum_{s, t<C} a_{i j s t} x^{j(p-1)+s k+t l}
$$

where $A, B, C$ are integer parameters which will be chosen to satisfy

$$
\begin{gathered}
A, B, C \geq 1, \\
C k \leq \frac{1}{2}(p-1), \\
B C^{2} \leq \delta_{ \pm}, \\
A \delta_{ \pm} \leq(p-1),
\end{gathered}
$$




$$
\begin{gathered}
B \leq \frac{k \ell_{ \pm}}{d_{1}} \frac{A}{(p-1)}, \\
D(C+D)^{2} t<A B C^{2} .
\end{gathered}
$$

Our goal is to show the existence of a set of coefficients $a_{i j s t} \in \mathbb{Z}_{p}$ such that $F$ is a non-zero polynomial mod $p$ with a high order zero at each $x$ counted in $C_{ \pm}\left(\mathbf{u}_{1}\right), \ldots, C_{ \pm}\left(\mathbf{u}_{t}\right)$.

The monomials in $f_{i}(x)$ will be distinct since if $j(p-1)+s k+t l=j^{\prime}(p-1)+s^{\prime} k+t^{\prime} l$ then from (3.5)

$$
\left|j-j^{\prime}\right|(p-1)=\left|\left(s-s^{\prime}\right) k+\left(t-t^{\prime}\right) l\right|<C k+C l<(p-1)
$$

and $j=j^{\prime}$, while from (3.6) we have $C<\left(k / d_{1}\right)$ and

$$
k\left(s-s^{\prime}\right)=l\left(t^{\prime}-t\right) \Rightarrow\left(k / d_{1}\right) \mid\left(t^{\prime}-t\right) \Rightarrow t=t^{\prime}, s=s^{\prime} .
$$

In [4] we showed that if $f(x)=x^{k_{1}} g_{1}(x)^{p}+\cdots+x^{k_{r}} g_{r}(x)^{p}$ in $\mathbb{Z}_{p}[x]$ with the $k_{i}$ distinct mod $p$, and $f(x)$ has a zero $a \neq 0$ of multiplicity $\nu$, then $\nu=w p+u$ with $u<r$ and $w$ the highest power of $(x-a)$ dividing all the $g_{i}$. In particular if $(x-1)^{\mu_{i}}|| f_{i}$ then $\mu_{i}=u_{i}+p v_{i}$ for some $u_{i}, v_{i}$ with $u_{i}<B C^{2}$. Hence the power of $(x-1)$ dividing the different $\Phi_{i} f_{i}$ will be distinct, since if

$$
i \delta_{ \pm}+u_{i}+p v_{i}=i^{\prime} \delta_{ \pm}+u_{i^{\prime}}+p v_{i^{\prime}}
$$

then, by (3.6) and (3.7)

$$
p\left|v_{i}-v_{i^{\prime}}\right|=\left|\left(u_{i}-u_{i^{\prime}}\right)+\left(i-i^{\prime}\right) \delta_{ \pm}\right|<B C^{2}+(A-1) \delta_{ \pm} \leq A \delta_{ \pm}<p,
$$

and $v_{i}=v_{i^{\prime}}$, while by $(3.6)$

$$
\left|i-i^{\prime}\right| \delta_{ \pm}=\left|u_{i}-u_{i^{\prime}}\right|<B C^{2} \leq \delta_{ \pm}
$$

gives $i=i^{\prime}$. Hence $F$ is not the zero polynomial in $\mathbb{Z}_{p}[x]$ unless all the $a_{i j s t}=0$.

Suppose that $a$ is counted in $C_{+}\left(u_{1}, u_{2}\right)$. Since $u_{1}, u_{2} \neq 0$ we have $a^{k}-1, a^{l}-1, a \neq 0$, and for a positive integer $D<p$, writing

$$
\nabla=x\left(x^{k}-1\right)\left(x^{l}-1\right) \frac{d}{d x},
$$

the polynomial $F(x)$ has a zero of order at least $D$ at $x=a$ iff

$$
\left.\left(x^{k}-1\right)^{-l A / d_{1}} \nabla^{r} F(x)\right|_{x=a}=0, \quad r=0, \ldots, D-1 .
$$

For $a$ counted in $C_{-}\left(u_{1}, u_{2}\right)$ we use instead

$$
\left.x^{-k l A / d_{1}} \nabla^{r} F(x)\right|_{x=a}=0, \quad r=0, \ldots, D-1 .
$$


Of course

$$
\begin{aligned}
\nabla x^{j} & =x^{j} j\left(x^{k}-1\right)\left(x^{l}-1\right), \\
\nabla\left(x^{l}-1\right)^{j} & =\left(x^{l}-1\right)^{j} j l x^{l}\left(x^{k}-1\right), \\
\nabla\left(x^{k}-1\right)^{j} & =\left(x^{k}-1\right)^{j} j k x^{k}\left(x^{l}-1\right) .
\end{aligned}
$$

Hence

$$
\nabla^{r} F(x)=\sum_{i<A} \sum_{j<B} \Phi_{i}(x) x^{j(p-1)} P_{i j r}\left(x^{k}, x^{l}\right)
$$

where the $P_{i j r}(x, y)$ are polynomials of degree at most $(C-1+r)$ in $x$ and $y$ whose coefficients will be linear expressions in the original $a_{i j s t}$. Since for $a$ counted in $C_{+}\left(u_{1}, u_{2}\right)$ the values

$$
\left(a^{k}-1\right)^{-l A / d_{1}} \Phi_{i}(a) a^{j(p-1)}=\left(u_{2}^{k / d_{1}} / u_{1}^{l / d_{1}}\right)^{i}
$$

and for $a$ counted in $C_{-}\left(u_{1}, u_{2}\right)$ the values

$$
a^{-k l A / d_{1}} \Phi_{i}(a) a^{j(p-1)}=\left((-1)^{k / d_{1}} u_{1}^{l / d_{1}} u_{2}^{k / d_{1}}\right)^{i}
$$

depend on $\mathbf{u}$ rather than $a$, we can make $\left(x^{k}-1\right)^{-l A / d_{1}} \nabla^{r} F(x)$ or $x^{-k l A / d_{1}} \nabla^{r} F(x)$ zero for each $a$ counted in $C_{+}(\mathbf{u})$ or $C_{-}(\mathbf{u})$ by making an appropriate polynomial $P_{r, \mathbf{u}}\left(x^{k}, x^{l}\right)$ of degree at most $(C-1+r)$ in $x^{k}$ and $x^{l}$, identically zero $\bmod p$; a requirement imposing $(C+r)^{2}$ homogeneous linear conditions on the $a_{i j s t}$. Thus for $F(x)$ to have a zero of order $D$ for each element counted in $C_{ \pm}(\mathbf{u})$ we need at most $D(C+D)^{2}$ linear equations, and for an integer $t \geq 1$, with $t D(C+D)^{2}$ equations we can ensure that $F$ has a root of order at least $D$ for each element counted in the $C_{ \pm}\left(\mathbf{u}_{1}\right), \ldots, C_{ \pm}\left(\mathbf{u}_{t}\right)$. Since the number of variables $a_{i j s t}$ is $A B C^{2}$, and elements counted in different $C_{ \pm}\left(\mathbf{u}_{i}\right)$ are distinct, condition (3.9) ensures the existence of a non-zero polynomial $F(x)$ in $\mathbb{Z}_{p}[x]$ with $\sum_{i=1}^{t} C_{ \pm}\left(\mathbf{u}_{i}\right)$ zeros, each of order at least $D$. Hence, from (3.8) and (3.5)

$$
D \sum_{i=1}^{t} C_{ \pm}\left(\mathbf{u}_{i}\right) \leq \operatorname{deg}(F) \leq A\left(k \ell_{ \pm} / d_{1}\right)+(p-1)(B-1)+C k+C l \leq 2 A\left(k \ell_{ \pm} / d_{1}\right)
$$

and

$$
C_{ \pm}\left(\mathbf{u}_{t}\right) \leq t^{-1} \sum_{i=1}^{t} C_{ \pm}\left(\mathbf{u}_{i}\right) \leq \frac{2 A\left(k \ell_{ \pm} / d_{1}\right)}{D t}
$$


It remains to choose parameters satisfying conditions (3.4) through (3.9):

$$
\begin{aligned}
D=C & =\left[2^{-\frac{7}{5}} \frac{(p-1)^{\frac{1}{5}} \delta_{ \pm}^{\frac{2}{5}}}{\left(k \ell_{ \pm} / d_{1}\right)^{\frac{1}{5}} t^{\frac{1}{5}}}\right], \\
B & =\left\lfloor 2^{\frac{4}{5}} \frac{\left(k \ell_{ \pm} / d_{1}\right)^{\frac{2}{5}} t^{\frac{2}{5}} \delta_{ \pm}^{\frac{1}{5}}}{(p-1)^{\frac{2}{5}}}\right], \\
A & =\left[2^{\frac{4}{5}} \frac{(p-1)^{\frac{3}{5}} t^{\frac{2}{5}} \delta_{ \pm}^{\frac{1}{5}}}{\left(k \ell_{ \pm} / d_{1}\right)^{\frac{3}{5}}}\right] .
\end{aligned}
$$

These choices ensure restrictions (3.6), (3.8) and (3.9) (the latter simplifies to $4 C t<A B$ ), and (3.10) gives the stated bound

$$
C_{ \pm}\left(\mathbf{u}_{t}\right) \leq 2^{\frac{26}{5}}(p-1)^{\frac{2}{5}}\left(k \ell_{ \pm} / d_{1}\right)^{\frac{3}{5}} t^{-\frac{2}{5}} \delta_{ \pm}^{-\frac{1}{5}}
$$

For (3.4) observe that the trivial bounds (3.3) give $A \geq 2^{6}, B \geq 2^{6}$ respectively, while $C \geq 1$ requires

$$
t \leq 2^{-7}(p-1) \frac{\delta_{ \pm}^{2}}{\left(k \ell_{ \pm} / d_{1}\right)} .
$$

The restriction $k<2^{-5}(p-1)^{\frac{2}{3}} d_{1}^{\frac{1}{6}} \ell_{ \pm}^{\frac{1}{6}}$, and $\delta_{ \pm}<2 k / d_{1}$, ensure that

$$
C k<2^{-\frac{7}{5}} \frac{(p-1)^{\frac{1}{5}} \delta_{ \pm}^{\frac{2}{5}} k}{t^{\frac{1}{5}}\left(k \ell_{ \pm} / d_{1}\right)^{\frac{1}{5}}}<\frac{1}{2} \frac{(p-1)^{\frac{1}{5}} k^{\frac{6}{5}}}{t^{\frac{1}{5}} d_{1}^{\frac{1}{5}} \ell_{ \pm}^{\frac{1}{5}}}<\frac{1}{2^{7} t^{\frac{1}{5}}}(p-1)
$$

so condition (3.5) certainly holds. Finally condition (3.7) is satisfied

$$
A \delta_{ \pm}<2^{\frac{9}{5}} \frac{(p-1)^{\frac{3}{5}} t^{\frac{2}{5}} \delta_{ \pm}^{\frac{6}{5}}}{\left(k \ell_{ \pm} / d_{1}\right)^{\frac{3}{5}}} \leq(p-1)
$$

as long as

$$
t \leq 2^{-\frac{9}{2}}(p-1)\left(\frac{\left(k \ell_{ \pm} / d_{1}\right)}{\delta_{ \pm}^{2}}\right)^{\frac{3}{2}}
$$

Restriction (3.13) supercedes (3.12) iff $\left(k \ell_{ \pm} / d_{1}\right)<\frac{1}{2} \delta_{ \pm}^{2}$ and the cutoff values $T$ are plain.

Notice that for $\left(k \ell_{ \pm} / d_{1}\right)<\frac{1}{2} \delta_{ \pm}^{2}$ and $k<2^{-5}(p-1)^{\frac{2}{3}} d_{1}^{\frac{1}{6}} \ell_{ \pm}^{\frac{1}{6}}$ we have, using $\delta_{ \pm}<2 k / d_{1}$,

$$
T=\left\lfloor 2^{-\frac{9}{2}}(p-1)\left(\frac{\left(k \ell_{ \pm} / d_{1}\right)}{\delta_{ \pm}^{2}}\right)^{\frac{3}{2}}\right\rfloor \geq\left\lfloor 2^{-\frac{15}{2}}(p-1) \frac{\ell_{ \pm}^{\frac{3}{2}} d_{1}^{\frac{3}{2}}}{k^{\frac{3}{2}}}\right\rfloor \geq\left\lfloor\ell_{ \pm}^{\frac{5}{4}} d_{1}^{\frac{5}{4}}\right\rfloor \geq 1
$$

and the bound (3.11) gives $C\left(\mathbf{u}_{T}\right) \leq 2^{\frac{37}{5}} \delta_{ \pm}$.

Corollary 3.1. For $k<2^{-5}(p-1)^{\frac{2}{3}} d_{1}^{\frac{1}{6}} l^{\frac{1}{6}}$,

$$
\sum_{i=1}^{N} C_{ \pm}^{2}\left(\mathbf{u}_{i}\right) \ll \max \left\{\delta_{ \pm},\left(k l / d_{1}\right) \delta_{ \pm}^{-\frac{1}{3}}\right\} p
$$


Proof. We assume that $k<2^{-5}(p-1)^{\frac{2}{3}} d_{1}^{\frac{1}{6}} l^{\frac{1}{6}}$. When

$$
L=\left\lfloor 2^{-7}(p-1) \frac{\delta_{ \pm}^{\frac{1}{3}}}{\left(k \ell_{ \pm} / d_{1}\right)}\right\rfloor \leq T
$$

Lemma 3.1 and $(2.2)$ give

$$
\begin{aligned}
\sum_{i=1}^{N} C_{ \pm}^{2}\left(\mathbf{u}_{i}\right) & \ll(p-1)^{\frac{4}{5}}\left(k l / d_{1}\right)^{\frac{6}{5}} \delta_{ \pm}^{-\frac{2}{5}} \sum_{t \leq L} t^{-\frac{4}{5}}+(p-1)^{\frac{2}{5}}\left(k l / d_{1}\right)^{\frac{3}{5}} \delta_{ \pm}^{-\frac{1}{5}} L^{-\frac{2}{5}} \sum_{t>L} C\left(\mathbf{u}_{t}\right) \\
& \ll(p-1)^{\frac{4}{5}}\left(k l / d_{1}\right)^{\frac{6}{5}} \delta_{ \pm}^{-\frac{2}{5}} L^{\frac{1}{5}}+(p-1)^{\frac{2}{5}}\left(k l / d_{1}\right)^{\frac{3}{5}} \delta_{ \pm}^{-\frac{1}{5}} L^{-\frac{2}{5}}(p-1) \\
& \ll(p-1)\left(k l / d_{1}\right) \delta_{ \pm}^{-\frac{1}{3}} .
\end{aligned}
$$

Clearly $L \leq T=\left\lfloor 2^{-7}(p-1) \frac{\delta_{ \pm}^{2}}{\left(k \ell_{ \pm} / d_{1}\right)}\right\rfloor$ when $\left(k \ell_{ \pm} / d_{1}\right) \geq \frac{1}{2} \delta_{ \pm}^{2}$, while when $\left(k \ell_{ \pm} / d_{1}\right) \leq$ $\frac{1}{2} \delta_{ \pm}^{2}$ we still have $L \leq T=\left\lfloor 2^{-\frac{9}{2}}(p-1)\left(\frac{k \ell_{ \pm}}{d_{1}}\right)^{\frac{3}{2}} \delta_{ \pm}^{-3}\right\rfloor$ if $\left(k \ell_{ \pm} / d_{1}\right)>\frac{1}{2} \delta_{ \pm}^{\frac{4}{3}}$.

When $\left(k \ell_{ \pm} / d_{1}\right)<\frac{1}{2} \delta_{ \pm}^{\frac{4}{3}}$ we use the bound $C_{ \pm}\left(\mathbf{u}_{T}\right) \leq 2^{\frac{37}{5}} \delta_{ \pm}$to obtain

$$
\begin{aligned}
\sum_{i=1}^{N} C_{ \pm}^{2}\left(\mathbf{u}_{i}\right) & \ll(p-1)^{\frac{4}{5}}\left(k l / d_{1}\right)^{\frac{6}{5}} \delta_{ \pm}^{-\frac{2}{5}} \sum_{t \leq T} t^{-\frac{4}{5}}+\delta_{ \pm} \sum_{t>T} C\left(\mathbf{u}_{t}\right) \\
& \ll(p-1)^{\frac{4}{5}}\left(k l / d_{1}\right)^{\frac{6}{5}} \delta_{ \pm}^{-\frac{2}{5}} T^{\frac{1}{5}}+\delta_{ \pm}(p-1) \\
& \ll(p-1) \frac{\left(k l / d_{1}\right)^{\frac{3}{2}}}{\delta_{ \pm}}+\delta_{ \pm}(p-1) \ll \delta_{ \pm}(p-1) .
\end{aligned}
$$

\section{Completion of the proof of Lemma 1.1}

Assume that $k<\frac{1}{32}(p-1)^{\frac{2}{3}} d^{\frac{1}{3}}$. Since $d \leq d_{1}$ plainly $d \delta_{ \pm}<2 k$ and $\left(d / d_{1}\right) \delta_{ \pm}^{-\frac{1}{3}} \leq \Delta_{ \pm}^{-\frac{1}{3}}$. So, from Corollary 3.1 and (2.1), we have

$$
M_{ \pm} \ll d^{2} p^{2}+k^{2} l p+\max \left\{k, k l \Delta_{ \pm}^{-\frac{1}{3}}\right\} p^{2}
$$

Clearly

$$
d^{2} p^{2}=k l \Delta_{ \pm}^{-\frac{1}{3}} p^{2}\left(\frac{d^{2}}{k l} \Delta_{ \pm}^{\frac{1}{3}}\right)<k l \Delta_{ \pm}^{-\frac{1}{3}} p^{2}\left(2^{\frac{1}{3}}(d / l)(d / k)^{\frac{2}{3}}\right)<k l \Delta_{ \pm}^{-\frac{1}{3}} p^{2}
$$

and

$$
k^{2} l p=k l \Delta_{ \pm}^{-\frac{1}{3}} p^{2}\left(\frac{k \Delta_{ \pm}^{\frac{1}{3}}}{p}\right)<k l \Delta_{ \pm}^{-\frac{1}{3}} p^{2}\left(\frac{2^{\frac{1}{3}} k^{\frac{4}{3}}}{d^{\frac{1}{3}} p}\right)<k l \Delta_{ \pm}^{-\frac{1}{3}} p^{2}\left(\frac{d^{\frac{1}{9}}}{p^{\frac{1}{9}}}\right)<k l \Delta_{ \pm}^{-\frac{1}{3}} p^{2} .
$$

Hence as claimed

$$
M_{ \pm} \ll \max \left\{k, k l \Delta_{ \pm}^{-\frac{1}{3}}\right\} p^{2} .
$$




\section{Proof of Proposition 1.1}

We use the technique of Akulinichev to average over the $d_{ \pm}^{*}$ th roots of unity. For any $f$ and $\chi$ we have

$$
\begin{aligned}
(p-1) S_{ \pm} & =\sum_{y=1}^{p-1} \chi\left(x y^{\frac{(p-1)}{d_{ \pm}^{*}}}\right) \sum_{x=1}^{p-1} e_{p}\left(f\left(x y^{\frac{(p-1)}{d_{ \pm}^{*}}}\right)\right) \\
& =\sum_{x=1}^{p-1} \chi(x) \sum_{y=1}^{p-1} \chi(y)^{\frac{(p-1)}{d_{ \pm}^{*}}} e_{p}\left(\left(a x^{k}+b x^{ \pm l}\right) y^{\frac{l(p-1)}{d_{ \pm}^{*}}}\right) .
\end{aligned}
$$

If $a x^{k}+b x^{ \pm l} \neq 0$ then Weil's bound gives

$$
\left|\sum_{y=1}^{p-1} \chi(y)^{\frac{(p-1)}{d_{ \pm}^{*}}} e_{p}\left(\left(a x^{k}+b x^{ \pm l}\right) y^{\frac{l(p-1)}{d_{ \pm}^{*}}}\right)\right| \leq\left(\frac{l(p-1)}{d_{ \pm}^{*}}, p-1\right) p^{\frac{1}{2}}=\frac{d(p-1)}{d_{ \pm}^{*}} p^{\frac{1}{2}}
$$

If $-b \bar{a}=\alpha^{(k \mp l)}$ for some $\alpha$ in $\mathbb{Z}_{p}^{*}$ then we also have the $d_{ \pm}^{*}$ values of $x$ with $a x^{k}+b x^{ \pm l}=$ 0 . If $\chi^{(p-1) / d_{ \pm}^{*}} \neq \chi_{0}$ then for these values of $x$ the inner sum $\sum_{y=1}^{p-1} \chi(y)^{\frac{(p-1)}{d_{ \pm}^{*}}}$ is zero, while if $\chi^{(p-1) / d_{ \pm}^{*}}=\chi_{0}$ this sum is $(p-1)$ and $\chi(x)=\chi(\alpha)$.

\section{Proof of Corollary 1.1}

First we show that if $d_{ \pm}^{*}<\frac{1}{4} p^{\frac{8}{13}} / d^{\frac{5}{13}}$, then uniformly

$$
\left|S_{ \pm}\right| \ll d^{\frac{3}{13}} p^{\frac{51}{52}} .
$$

The strategy is to make an appropriate change of variables of the type $x \rightarrow x^{m}$ so that Theorem 1.1 can be applied effectively. ¿From the box principle there exists an integer $m$ such that

$$
m k \equiv \alpha \quad \bmod (p-1), \quad \pm m l \equiv \beta \quad \bmod (p-1),
$$

(plus sign for $S_{+}$and minus for $S_{-}$) with

$$
|\alpha| \leq 2(p d)^{\frac{8}{13}}, \quad|\beta| \leq 2(p d)^{\frac{5}{13}},
$$

and $m \not \equiv 0 \bmod (p-1) / d$ (so that $\alpha, \beta$ are not both 0$)$. We can assume that $2(p d)^{\frac{8}{13}}<$ $\frac{1}{32}(p-1)^{\frac{2}{3}}$, else $d^{\frac{3}{13}} p^{\frac{51}{52}} \gg p$ and the bound is trivial.

Suppose that $(m, p-1)=\nu$ and write $\mathbb{Z}_{p}^{*} /\left(\mathbb{Z}_{p}^{*}\right)^{m}=\left\{w_{1}, \ldots, w_{\nu}\right\}$ so that

$$
S_{ \pm}=\sum_{i=1}^{\nu} \frac{\chi\left(w_{i}\right)}{\nu} \sum_{x=1}^{p-1} \chi(x)^{m} e_{p}\left(a w_{i}^{k} x^{\alpha}+b w_{i}^{ \pm l} x^{\beta}\right) .
$$

If $\beta=0$ (or similarly $\alpha=0$ ) then applying the Heath-Brown \& Konyagin bound (1.4) to the inner sum gives

$$
\left|S_{ \pm}\right| \ll|\alpha|^{\frac{3}{8}} p^{\frac{3}{4}} \ll d^{\frac{3}{13}} p^{\frac{51}{52}}
$$

(strictly speaking their result does not contain a $\chi$ but it is easily seen that Lemma 1 of [5], and hence the result, still holds with the addition of a multiplicative character). 
So we can assume that $\alpha, \beta \neq 0$ and, since $d_{ \pm}^{*}<\frac{1}{4} p^{\frac{8}{13}} / d^{\frac{5}{13}}$ ensures that $|\beta|<(p-1) / d_{ \pm}^{*}$ (and hence $\left.m \not \equiv 0 \bmod (p-1) / d_{ \pm}^{*}\right)$, that $\alpha \not \equiv \beta \bmod (p-1)$. If $|\alpha|<2|\beta|$ then by the Mordell bound (1.8)

$$
\left|S_{ \pm}\right| \ll(|\alpha||\beta|)^{\frac{1}{4}} p^{\frac{3}{4}} \ll|\beta|^{\frac{1}{2}} p^{\frac{3}{4}} \ll d^{\frac{5}{26}} p^{\frac{49}{52}}
$$

So we can assume that $0<|\beta|<\frac{1}{2}|\alpha|$, with $|\alpha|<\frac{1}{32}(p-1)^{\frac{2}{3}}$. Hence

$$
\Delta_{ \pm}=\frac{|\alpha| \mp|\beta|}{(\alpha, \beta, p-1)}>\frac{1}{2} \frac{|\alpha|}{|\beta|}
$$

and from Theorem 1.1,

$$
\begin{aligned}
\left|S_{ \pm}\right| \ll \max \left\{1,|\beta|(|\alpha| /|\beta|)^{-\frac{1}{3}}\right\}^{\frac{1}{4}}|\alpha|^{\frac{1}{4}} p^{\frac{3}{4}}=\max \left\{|\alpha|^{\frac{1}{4}},|\alpha|^{\frac{1}{6}}|\beta|^{\frac{1}{3}}\right\} p^{\frac{3}{4}} \\
\ll \max \left\{d^{\frac{2}{13}} p^{\frac{2}{13}}, d^{\frac{3}{13}} p^{\frac{3}{13}}\right\} p^{\frac{3}{4}} \ll d^{\frac{3}{13}} p^{\frac{51}{52}}
\end{aligned}
$$

Suppose now that $d_{ \pm}^{*}>\frac{1}{4} p^{\frac{8}{13}} / d^{\frac{5}{13}}$. We may also assume $d<p^{\frac{1}{12}}$ for otherwise $(6.1)$ is trivial. Then

$$
\frac{d}{d_{ \pm}^{*}} p^{\frac{3}{2}}<4 d^{\frac{18}{13}} p^{\frac{23}{26}}=4 d^{\frac{3}{13}} d^{\frac{15}{13}} p^{\frac{23}{26}}<4 d^{\frac{3}{13}} p^{\frac{51}{52}}
$$

The corollary follows immediately from Proposition 1.1.

\section{REFERENCES}

[1] N. M. Akulinichev, Estimates for rational trigonometric sums of a special type, Doklady Acad. Sci. USSR 161 (1965), 743-745. English trans. in Doklady 161, no. 4 (1965), 480-482.

[2] T. Cochrane \& C. Pinner, An improved Mordell type bound for exponential sums, to appear in Proc. Amer. Math. Soc.

[3] T. Cochrane, C. Pinner \& J. Rosenhouse, Bounds on exponential sums and the polynomial Waring's problem mod $p$, to appear in J. London Math. Soc.

[4] _ Sparse polynomial exponential sums, to appear in Acta. Arith.

[5] D. R. Heath-Brown \& S. Konyagin, New bounds for Gauss sums derived from kth powers, and for Heilbronn's exponential sum, Quart. J. Math. 51 (2000), 221-235.

[6] A. A. Karatsuba, On estimates of complete trigonometric sums, Matem. Zametki. 1 (1967), 199-208 (Russian); translation in Math. Notes (1968), 133-139.

[7] L. J. Mordell, On a sum analagous to a Gauss's sum, Quart. J. Math. 3 (1932), 161-167.

[8] G. I. Perel'muter, Estimate of a sum along an algebraic curve, (Russian), Mat. Zametki 5 (1969), 373-380.

[9] A. Weil, On some exponential sums, Proc. Nat. Acad. Sci. U.S.A. 34 (1948), 204-207.

[10] H. Yu, Estimates for complete exponential sums of special types, Math. Proc. Camb. Phil. Soc. 131 (2001), 321-326. 
Department of Mathematics, Kansas State University, Manhattan, KS 66506

E-mail address: cochrane@math.ksu.edu

Department of Mathematics, Kansas State University, Manhattan, KS 66506

E-mail address: pinner@math.ksu.edu 